\title{
Assessment of fully vaccination coverage and associated factors among children aged 12-23 months in Mecha district, North West Ethiopia: A cross-sectional study
}

\author{
Ayal Debie ${ }^{1, *}$, Bekele Taye ${ }^{2}$ \\ ${ }^{1}$ Department of Public Health, Bahirdar Private Clinic, Bahirdar, Ethiopia \\ ${ }^{2}$ Department of Public Health Health, College of Medicine and Health Sciences, Bahirdar University, Bahirdar, Ethiopia
}

\section{Email address:}

debieayal@gmail.com(A. Debie), bekeletaye414@gmail.com (B. Taye)

\section{To cite this article:}

Ayal Debie, Bekele Taye. Assessment of Fully Vaccination Coverage and Associated Factors among Children Aged 12-23 Months in Mecha District, North West Ethiopia: A Cross-Sectional Study. Science Journal of Public Health. Vol. 2, No. 4, 2014 , pp. 342-348. doi: $10.11648 /$ j.sjph.20140204.26

\begin{abstract}
Background: About $85 \%$ of the world's children under one year of age have received life-saving vaccines. An estimated 1.7 million children died in 2008 from vaccine-preventable diseases and in 2011, only $24 \%$ of the children were fully vaccinated. Objectives: To assess fully vaccination coverage and associated factors among children aged 12-23 months in Mecha district. Methods: A cross-sectional community-based study was conducted in 8 rural and 1 urban kebeles from April to October, 2013. A systematic sampling method was used for sample selection. Data were collected on 497 children aged 12-23 months from 497 representative households were collected using trained nurses. The data collectors assessed collected the vaccination status of the children based on vaccination cards or mother's verbal reports using a pre-tested semi-structured interviewer administered questionnaire through house-to-house visits. Data were coded and entered into SPSS 20.0 versions. Bivariate and multivariate logistic regression analyses were used to assess factors associated with vaccination status of children. Those predictor variables with $\mathrm{p}$-value $<0.05$ were considered significantly associated with the outcome variable. Results: About $49.3 \%$ of children aged 12-23 months were fully vaccinated by card and mother's recall, and $1.6 \%$ children were not started vaccination. Using multivariate logistic regression models, factors significantly associated with fully vaccination were urban residence (AOR :2.401, 95\% CI:1.011,5.705), mothers who have good knowledge on vaccination (AOR: 2.131, 95\% CI:1.076, 4.217), taking the child to health institution during illness in the first year of life (AOR: 2.147 , 95\% CI: 1.275, 3.615), institutional delivery (AOR :1.593, 95\% CI :1.037,2.445) and complete primary \& above education of father of the child( AOR:1.814, 95\% CI:1.008,3.265). Conclusion and recommendation: Complete vaccination coverage among children aged 12-23 months remains very low in the district. Health institution delivery , urban residence, mother's knowledge on vaccination and father complete primary and above level of education were found to be independent factors for fully child vaccination status of children in the district. Therefore both HEWs and other staff members of health centres should be improve awareness of the people by designing proper health education targeting the mother on vaccination.
\end{abstract}

Keyword: Fully Vaccination, Mecha District, Amhara Region

\section{Introduction}

Discovery of vaccine is one of the successes of public health; it has been an instrument for saving millions of lives. About $85 \%$ of the world's children under one year of age have received life saving vaccines. Whereas, one fifth of the world's children about 19.3 million infants were not immunized against vaccine preventable childhood killer diseases. Nearly $70 \%$ of these children live in ten countries. An estimated 1.7 million children died in 2008 from vaccine preventable diseases. The deadlines for eliminating maternal and neonatal tetanus and certification of global polio eradication by 2010 have not been met (1).

Although estimated global routine measles vaccination 
coverage reached $82 \%$ in 2007 , nearly 23.2 million children were unvaccinated of which 15.3 million $(65 \%)$ resides in eight countries mainly in Africa (2).

All countries that routinely provide a second dose of measles vaccine achieve 90\% 2 dose measles containing vaccination $(\mathrm{MCV})$ coverage nationally and at least $80 \%$ coverage in every district and have case based measles surveillance and report at least 2 non measles cases per 100,000 population. At the end of 2015, reduce estimated global measles mortality by $95 \%$ or more in comparison with 2000 (3).

Immunization is one of the national child survival strategies in the country to reach diphtheria-pertusis and tetanus (DPT3) and measles vaccination coverage 90\% in 2010 (4).According to demographic health survey(EDHS) report among children age 12-23 months $24 \%$ were fully vaccinated , $66 \%$ of children had received the BCG vaccine and $56 \%$ had received the measles vaccine (5).

However, this national or regional data may not reflect the situation of specific districts. Factors may also vary from district to district. so this study is designed to show the local situation.

\section{Methods}

\subsection{Study Setting}

A community based cross sectional study was employed using quantitative method of data collection in Mecha district, Western Amhara from April 2013 to October, 2013. The district is one of the 15 woredas of West Gojjam Zone in Amhara regional state. It is located $35 \mathrm{kms}$ South West of Bahir Dar on the main road of Bahir Dar to Addis Ababa. The total population of the district is about 323,950. Of the total population about $161003(49.7 \%)$ are males and $162947(50.3 \%)$ are females. The district has 40 rural and 4 urban Kebeles (the smallest administrative unit). The district has a total of 10 health centre and 40 health posts. There are five NGOs working maternal and new born health area in the district.

\subsection{Sampling}

The source population of the study was all children aged 12 to 23 months in the district. The study population were all children aged 12 to 23 months in the selected kebeles of the district .Sample size was calculated using a single population proportion formula assuming proportion of fully immunization is $\mathrm{p}(26.7 \%)$ from research conducted across regional states on 2011 fully immunization coverage in Amhara region [5 ], 5\% margin of error (d) and 95\% (za/2 = 1.96) confidence level, then after considering design effect 1.5 and non response rate of $10 \%$, the final sample size was 497.

\subsection{Sampling Technique}

A multi stage stratified sampling technique was used with the strata of kebeles. A total of 9 kebeles, one from urban and eight from rural were randomly selected and included in the study .The total sample size was allocated proportionally to each kebele based on the number of children age 12 to 23 months. Then respondents were selected by systematic random sampling from health extension workers registration book. Data were collected using structured Amharic questionnaire through interview by trained nurses supervised by health officers. The questionnaire was adopted from literature review $(5,13)$ and comprises four sections: socio-demographic characteristics of participants, reproductive history, Vaccination status of children by history or card, and Knowledge of mother's on vaccination. In this study, when a child is considered as fully vaccinated when BCG, three doses of PENTA, three doses of OPV, three doses of PCV and measles vaccine are recorded.

Vaccinated by card only refers to when child vaccination data were collected from children vaccination card only. Vaccinated by history only refers to when child vaccination data collected from child mother or care taker by history only.

Knowledge: mothers who answered mean and above on knowledge questions is referred to as having good knowledge where as answered below the mean considered to be having poor knowledge.

A structured questionnaire was prepared in English and translated into Amharic language and was re-translated back to English by Linguistic graduates and Health professionals to ensure consistency. A one day training regarding the objectives of the study and ways of administering the questionnaire was given to the data collectors as well as the supervisors by the investigator. The prepared questionnaire was pre-tested prior to the actual data collection on $5 \%$ of sample size participants that were not included in the main survey.

The data were coded and entered into SPSS 20.0 version.

\subsection{Data Analysis}

Bivarate and multivariable analyses were computed to test whether there was association between fully vaccination and selected independent variables.

\subsection{Ethical Consideration}

Ethical clearance was obtained from ethical review committee of GAMBY College of medical science. Letter of permission was obtained from the health bureau and Mecha district health office. The confidentiality of information was maintained by excluding personal identifiers and interview privately. Data were collected after securing informed consent from every respondent..

\section{Results}

\subsection{Socio Demographic Characteristics of Participant}

A total of 497 mothers of children age between 12-23 months were interviewed about immunization with a response rate of $100 \%$.Out of 497 children 257 (51.7\%) were males. The mean age for children were 17.5 
months .About 461(92.8\%) children live in rural area. The mean age for the mother was 27.6 years. Only $34(6.8 \%)$ and $30(6 \%)$ of mothers/guardians, $44(8.9 \%)$ and $35(7 \%)$ of father of children attended primary and secondary education, respectively. Apart from this, out of 497 mothers/guardians of children orthodox (98.2\%), farmer (75.7\%), merchant $(12.3 \%)$, married $(92.8 \%)$ and having secondary and above educated children in the house hold (19.1\%) (Table 1).

\subsection{Vaccination Status of Children by History or Card in Mecha District}

Out of the total surveyed children aged 12-23 months, Only 245(49.3\%) of children completed all the recommended vaccines by history and card, but about 373(75.1\%) children were fully vaccinated by only history. Whereas partially vaccinated by card were only $78(15.7 \%) \&$ based on maternal recalling on children partially vaccinated were only $116(23.3 \%)$ received one or more of the nine recommended vaccines and $8(1.6 \%)$ have never been vaccinated.

Vaccination coverage by history, OPV was the most frequently received vaccine. Particularly, OPV1 and PENTA1 were taken by the majority of children 489 (98.4\%) followed by PCV1 487 (98\%) and BCG 485(97.6\%). OPV0 $(58.4 \%)$ and measles (79\%) coverage were the least taken vaccines. PENTA dropout rate was $13.9 \%$ and BCG to measles vaccine dropout rate was $18.6 \%$. The coverage showed a decrement from the first doses of vaccine to the last doses.

Total coverage by card was calculated by taking children who had vaccination card as a numerator. From the total 497 children 323(65\%) took OPV1 and PENTA1 followed by BCG 322(64.8\%). PCV1 was also taken by $321(64.6 \%)$ of children.OPV0 (39\%) and measles 266(53.5\%) were the least taken vaccine by children. BCG to measles vaccine dropout rate was $11.3 \%$ (Figure 1).

Table 1. Socio-demographic characteristics and fully vaccination coverage among children aged 12-23 months in Mecha district, 2013. (N=497).

\begin{tabular}{|c|c|c|c|c|c|}
\hline \multirow{2}{*}{ Variables } & \multirow{2}{*}{ Category } & \multicolumn{2}{|c|}{ Fully by card } & \multirow{2}{*}{ Frequency } & \multirow{2}{*}{ Percentage (\%) } \\
\hline & & Yes & No & & \\
\hline \multirow{2}{*}{ Sex of the child } & Male & 131 & 126 & 257 & 51.7 \\
\hline & Female & 114 & 126 & 240 & 48.3 \\
\hline \multirow{2}{*}{ Religion } & Orthodox & 238 & 250 & 488 & 98.2 \\
\hline & Muslim & 7 & 2 & 9 & 1.8 \\
\hline \multirow{4}{*}{ Marital status } & Married & 230 & 231 & 461 & 92.8 \\
\hline & Divorced & 7 & 11 & 18 & 3.6 \\
\hline & Separated & 2 & 6 & 8 & 1.6 \\
\hline & Widowed & 6 & 4 & 10 & 2 \\
\hline \multirow{6}{*}{$\begin{array}{l}\text { Age of mother/ } \\
\text { guardian in years }\end{array}$} & $15-19$ & 13 & 10 & 23 & 4.6 \\
\hline & $20-24$ & 60 & 73 & 133 & 26.8 \\
\hline & $25-29$ & 90 & 91 & 181 & 36.4 \\
\hline & $30-34$ & 42 & 42 & 84 & 16.9 \\
\hline & $35-40$ & 30 & 27 & 57 & 11.5 \\
\hline & $40+$ & 10 & 9 & 19 & 3.8 \\
\hline \multirow{2}{*}{ Residence } & Urban & 28 & 8 & 36 & 7.2 \\
\hline & Rural & 217 & 244 & 461 & 92.8 \\
\hline \multirow{3}{*}{ Maternal education } & Unable to read and write & 152 & 171 & 323 & 65 \\
\hline & Able to read and write & 52 & 58 & 110 & 22.1 \\
\hline & Complete primary\& above education & 41 & 23 & 64 & 12.8 \\
\hline \multirow{4}{*}{ Occupation } & House wife & 19 & 27 & 46 & 9.3 \\
\hline & Gov.t employee & 12 & 2 & 14 & 2.8 \\
\hline & Farmers & 180 & 196 & 376 & 75.7 \\
\hline & Merchant & 26 & 35 & 61 & 12.3 \\
\hline \multirow{3}{*}{ Father education } & Unable to read and write & 121 & 140 & 261 & 52.5 \\
\hline & Able to read and write & 69 & 88 & 157 & 31.6 \\
\hline & Com. primary and above education & 55 & 24 & 79 & 15.9 \\
\hline \multirow{2}{*}{ Educated children } & Yes & 47 & 48 & 95 & 19.1 \\
\hline & No & 198 & 204 & 402 & 80.9 \\
\hline
\end{tabular}




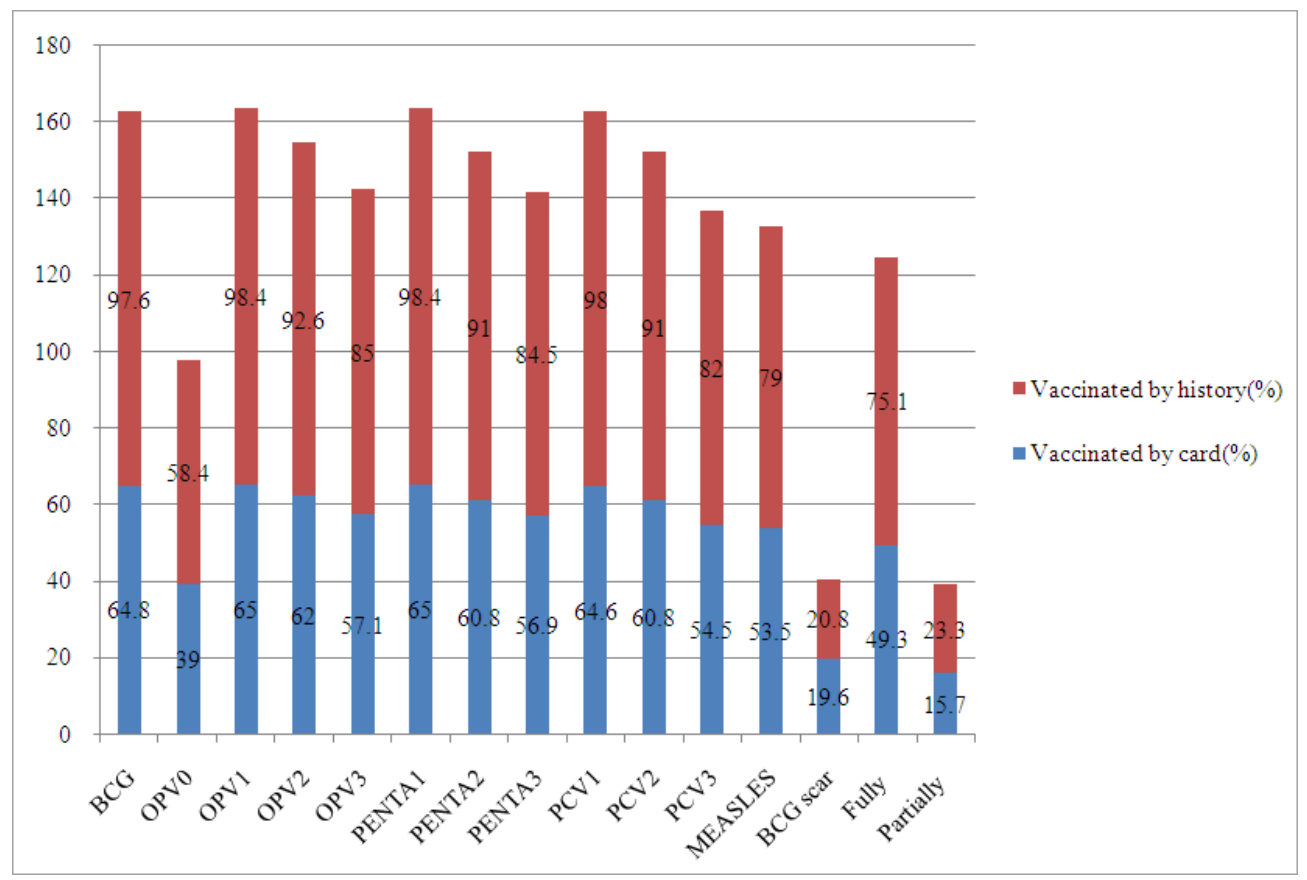

Figure 1. Vaccination status of children aged 12-23 months in Mecha district, 2013.

\subsection{Previous Obstetric History of Mothers of Children}

Out of the total mothers of children about 457 ( $92 \%$ ) had ANC follow up and also only $30.6 \%$ were delivered in the health facility .Beside to this, mother's/guardian's had 1-3 children 340 (68.4\%), still births(5.8\%) ,abortions(12.5\%) and took their child to health facility during illness in the $1^{\text {st }}$ year of life 416( 83.7\%) (Table 2).

\subsection{Knowledge of Mother's on Vaccination}

As described in the table, mothers of children who were heard about immunization 484(97.4\%), advantage of vaccination was for prevention of diseases 477 (96\%), age of children for starting vaccination at birth $352(70.8 \%$, age of children for completing vaccination $437(87.9 \%)$ and about $102(20.5 \%)$ of the respondents knew number of diseases prevented by immunizing the children (Table 3 )

Table 2. Frequency of previous obstetric history of mother's/guardians and fully vaccination coverage of children aged 12-23 months in Mecha district, 2013.

\begin{tabular}{|c|c|c|c|}
\hline Variables & Category & frequency & Percentage. (\%) \\
\hline \multirow{2}{*}{ ANC } & Yes & 457 & 92 \\
\hline & No & 40 & 8 \\
\hline \multirow{2}{*}{ Place of delivery } & At home & 345 & 69.4 \\
\hline & At health facility & 152 & 30.6 \\
\hline \multirow{2}{*}{$\begin{array}{l}\text { Number of alive } \\
\text { children }\end{array}$} & $1-3$ & 340 & 23.7 \\
\hline & $>=4$ & 157 & 67.8 \\
\hline \multirow{2}{*}{ Still birth } & Yes & 29 & 5.8 \\
\hline & No & 468 & 94.2 \\
\hline \multirow{2}{*}{ Abortion } & yes & 62 & 12.5 \\
\hline & No & 435 & 87.5 \\
\hline \multirow{2}{*}{ Lose pregnancy } & Yes & 82 & 16.5 \\
\hline & No & 415 & 83.5 \\
\hline Taking child to & Yes & 416 & 83.7 \\
\hline $\mathrm{h} / \mathrm{f}$ during illness & No & 81 & 16.3 \\
\hline
\end{tabular}

Table 3. Frequency of knowledge of mothers of children aged 12-23 months in Mecha district, 2013.

\begin{tabular}{llll}
\hline Variables & Category & Frequency & Percentage (\%) \\
\hline Heard about & Yes & 484 & 97.4 \\
immunization & No & 13 & 2.6 \\
Advantage of & D/se prevention & 477 & 96 \\
immunization & Benefit of HCP & 20 & 4 \\
Start age & Birth & 352 & 70.8 \\
& Six weeks & 145 & 29.2 \\
Age of completing & Nine & 437 & 87.9 \\
No of diseases & Twelve & 60 & 12.1 \\
prevented & Six & 395 & 79.5 \\
Knowledge & Nine & 102 & 20.5 \\
& Good & 451 & 90.7 \\
\hline
\end{tabular}

\subsection{Reasons for Non Vaccination}

In addition to listed factors, various reasons were described by the mothers/guardians for discontinuation or not started vaccination their children. These include fear of injections $72(14.5 \%)$, fear of side effects $67(13.5 \%)$, absence of vaccine $25(5 \%)$, negligence $19(3.8 \%)$, poor communication with mothers /guardians 16(3.2\%), far distance from health facility $2(0.4 \%)$, lack of information about the days for vaccination $9(1.8 \%)$ and poor neatness of the health facility $1(0.2 \%)$.

\subsection{Factors Associated with Fully Vaccination}

Multivariate backward stepwise analysis were conducted to assess the association between fully vaccination and factors associated with vaccination status of children.It showed that mother/guardian with urban residence (AOR :2.401, 95\% CI:1.011,5.705), mothers who have good knowledge on vaccination (AOR:2.131,95\%CI:1.076,4.217), 
taking the child to health institution during illness in the first year of life (AOR:2.147 ,95\%CI: 1.275, 3.615), institutional delivery (AOR :1.593,95\%CI:1.037,2.445) and complete primary \& above education of father of the child (AOR:1.814,95\%CI:1.008,3.265) were significantly associated with complete immunization status of children.

In addition to this, religion, level of education, occupation, marital status of mother/guardian, presence /absence of secondary and above educated children in the house hold ,ANC follow up, number of alive children ,still birth ,abortion ,knowledge on advantage of immunization, heard about immunization, age of the child for starting vaccination, knowing vaccine preventable diseases in number and profession of health worker who gave the vaccine for the children were not significantly associated with complete child immunization (Table 4).

\section{Discussion}

This study assessed fully vaccination coverage and factors associated with it among children aged between 12-23 months old in Mecha district of Amhara regional state. Based on vaccination card and history $49.3 \%$ children were fully vaccinated and $1.6 \%$ was never vaccinated.

Comparing the vaccination coverage of Mecha district with 2008 immunization coverage of the world the proportion of children fully vaccinated in the present study was less than by $35.7 \%$ and $28.5 \%$ from measles vaccination coverage $(1,2)$ This might be majority of the study population for the worlds data might be population of the developed countries.

Measles vaccination coverage in the present study was less than that of the strategic plan of CDC from 2011-2015 that is the proportion of children taking measles was less than by $26.5 \%$ (3). In addition to this, the current finding is lower than that of $\mathrm{FMOH}, 2005$ national child survival strategic plan by $30 \%$ and $36.5 \%$ from PENTA3 and measles vaccination coverage, respectively (4).

Table 4. Back ward stepwise multivariate analysis on associated factors for complete child vaccination coverage in Mecha district,2013 .

\begin{tabular}{|c|c|c|c|c|c|c|c|c|}
\hline \multirow{2}{*}{ Factors } & \multirow{2}{*}{ Category } & \multicolumn{2}{|c|}{ Fully by card } & \multirow{2}{*}{ Freq. } & \multirow{2}{*}{ COR } & \multirow{2}{*}{ AOR } & \multicolumn{2}{|c|}{$95 \% \mathrm{CI}$} \\
\hline & & Yes & No & & & & Lower & Upper \\
\hline \multirow{3}{*}{ Residence } & Urban & 28 & 8 & 36 & 3.94 & 2.401 & 1.011 & 5.705 \\
\hline & Rural & 217 & 244 & 461 & 1.00 & 1.00 & & \\
\hline & Education of father & & & & & & & \\
\hline \multirow{3}{*}{ Father education } & Unable to read and write & 121 & 140 & 261 & 1.00 & 1.00 & & \\
\hline & Able to read and write & 69 & 88 & 157 & 0.91 & 0.807 & 0.534 & 1.221 \\
\hline & Complete primary $\&$ above education & 55 & 24 & 79 & 3.085 & 1.814 & 1.008 & 3.265 \\
\hline \multirow{2}{*}{ Place of birth } & At home & 153 & 192 & 345 & 1.00 & 1.00 & & \\
\hline & At h/facility & 92 & 60 & 152 & 1.924 & 1.593 & 1.037 & 2.445 \\
\hline \multirow{2}{*}{ Taking child to $\mathrm{h} /$ facility } & Yes & 216 & 200 & 416 & 1.937 & 2.147 & 1.275 & 3.615 \\
\hline & No & 29 & 52 & 81 & 1.00 & 1.00 & & \\
\hline \multirow{2}{*}{ Knowledge } & Good & 232 & 219 & 451 & 2.689 & 2.131 & 1.076 & 4.217 \\
\hline & Poor & 13 & 33 & 46 & 1.00 & 1.00 & & \\
\hline
\end{tabular}

The proportion of fully vaccinated children in this study was about $29.3 \%$ and $25.3 \%$ higher than the EDHS 2005 and 2011 EPI survey coverage, respectively $(5,12$ ). This difference is because of the coverage at country level includes inaccessible area for immunization.

Apart from this ,comparing the vaccination coverage of Mecha district with immunization coverage survey conducted in Ambo district Oromia regional state ,2011, it is higher than by $14.3 \%(13)$. This difference might be catchment area of Mecha district is more accessible for vaccinating and getting information. But it is lower than the immunization coverage reported in the 2012 district health office report. This difference may be due to over reporting of the district health office.

From the total interviewed mothers of children, 323 (65\%) mothers showed the vaccination card of their children. From the card most children took PENT1 and OPV1 vaccines, but only $49.3 \%$ of finished the immunization without considering presence of BCG scar. The PENTA 3, PCV 3 and measles coverage were $56.9 \%, 54.5 \%$ and $53.5 \%$,respectively.The OPV3 vaccine coverage was slightly higher than the coverage of the PENTA3 and PCV3 vaccines. The measles coverage was less than PENTA3 and
PCV3 coverage. The reason might be the long time gap between the two vaccines in which the mothers may not return for measles vaccine.

Regarding knowledge of mothers about vaccination at which the child begins and finishes the immunization, $70.8 \%$ of the respondents knew the correct age at the child begins vaccination and $87.9 \%$ of them knew the correct age at which the child completes vaccination. The study also showed about $97.4 \%$ heard about immunization, 96\% knew the advantage of immunization was for prevention of diseases and $20.5 \%$ mothers/guardians knew number of vaccine preventable diseases. Those mothers who have good knowledge on vaccination were more likely fully vaccinated. Vaccination status of children whose mothers/guardians were urban residence more likely fully vaccinated than rural. This finding is consistence with the study done in India and Ambo district $(7,13)$. The explanation may be urban population had more accessible for information and getting services and mothers having knowledge on vaccination gave more attention.

However, in this study children delivered at health facilities were more likely to be complete their child's immunization than children delivered at home. This finding was similar with the study done in Ambo district (13). The 
explanation related to this may be mothers who gave birth at health institution were closer to the health service and getting information about immunization just after birth in the health institution.

In Ethiopia it was indicated that lack of awareness about immunization contribute to low immunization coverage. The findings of this study also showed that lack of knowledge on vaccination and fully immunization among children aged 12-23 months. Mothers of children those who had good knowledge on vaccination were more likely fully vaccinated than those who did not have. These finding is consistent with the study findings from Ambo and Wonago district in Ethiopia $(13,14)$. This may be those mothers who didn't know the correct age of completing vaccination had a chance to pass target ages of the child.

In this study mother's/guardian's of children who took their children to health facility within one year of life children during illness were more likely fully immunized. This result was consistent with the study finding from Wonago district south Ethiopia (14).This might be those children who took to health facility had a chance getting information on immunization and advice about childhood immunization.

In this study educational status of mother/guardian of children, marital status and presence / absence of secondary and above educated children in the household of the mothers did not show significant association with the completion of immunization among children aged between 12-23 months. This finding is similar with a study done in Ambo and Wonago district in Ethiopia $(13,14)$.

Even though, there was no significant association between children fully vaccination and profession of health care providers, children who were vaccinated by health centre provider became more likely develop BCG scar than vaccinated by HEWs. This might be skill differences on BCG administration and poor cold chain maintenance either at community level or during transportation of the vaccine from the health centre to health posts.

\section{Conclusions}

Complete vaccination coverage among children aged 12-23 months remains very low in the district. Place of delivery, level of education of fathers of children, taking children to health facility during illness in the $1^{\text {st }}$ year of life mother's knowledge on vaccination and place of residence of mothers were found to be independent factors affecting fully vaccination status of children in the district. Therefore both HEWs and other staff members of health centres should be improve awareness of the people by designing proper health education targeting the mother on vaccination. Health care providers should be mobilizing the community about the improvement of their service provision on institutional delivery in order to improve childhood vaccination. Further study should be conducted in order to identify factors associated with BCG scar as well as to ensure effect level of education of father of children on fully vaccination.

\section{Acronyms}

$\begin{array}{ll}\text { ANC } & \text { Ante Natal Care } \\ \text { BDU } & \text { Bahir Dar University } \\ \text { BCG } & \text { Bacillus Calmetee Gurian } \\ \text { CDC } & \text { Center of Disease control } \\ \text { DPT } & \text { Diphtheria, Pertusis and Tetanus } \\ \text { EDHS } & \text { Ethiopian Demographic Health Survey } \\ \text { FMOH } & \text { Federal Ministry of Health } \\ \text { GC } & \text { Gregorian Calendar } \\ \text { GCMS } & \text { GAMBY College of Medical Sciences } \\ \text { HEWs } & \text { Health Extension Workers } \\ \text { MD } & \text { Medical Doctor } \\ \text { MDG } & \text { Millennium Development Goals }\end{array}$

\section{Competing Interests}

The author(s) declared no competing interests.

\section{Authors' Contributions}

Ayal Debie designed the study, conducted field work, analyzed data, interpreted findings, and wrote the manuscript. Bekele Taye involved in the design, development of the proposal, assisted field work, in data analysis and manuscript writing. All authors of the manuscript have read and agreed to its content.

\section{Acknowledgements}

We are grateful to Bahir Dar University and GAMBY college of medical sciences for giving ethical aproval to conduct this research. We sincerely thank the study participants and data collectors of this study.

\section{References}

[1] UNICEF: Expanding immunization coverage,2010.

[2] WHO: Global elimination of measles, 2009. (source: IVB/measles model 2012) available from www.thelancet.com Published online April 24, 2012 DOI:10.1016/S0140-6736(12)60522-4

[3] Center of diseases control: Global immunization strategic framework, 2011-2015. Centers for Disease Control and Prevention 1600 Clifton Rd. Atlanta, GA 30333, USA 800-CDC-INFO (800-232-4636) TTY: (888) 232-6348.

[4] FMOH: Ethiopian child survival strategy department of family health, 2005.

[5] Central Statistical Agency Addis Ababa, Ethiopia ICF International Calverton, Maryland, USA March, 2012: Child vaccination coverage. Ethiopian demographic and Health Survey, 2011.

[6] WHO, UNICEF: Global immunization coverage data, July,2012 
[7] Phukan R, Barman M, Mahanta. Factors associated with immunization coverage of children in Assam, India: over the first year of life. J. Tro. Pediat,2009 Aug;55(4):249-52

[8] Charles S., Olalekan A, Olalekan A,etal. Individual and contextual factors associated with low Childhood immunization coverage in Sub-Saharan Africa.

[9] Uganda Bureau of Statistics (UBOS) and ICF International Inc. 2012: Child vaccination coverage. Uganda Demographic and Health Survey ,2011.

[10] Lilian C.,Simon K., Janeth K.,et al. Immunization coverage and its determinant among children aged $12-23$ months in a peri-urban area of Kenya. The Pan African Medical Journal. $2013 ; 14: 3$

[11] Central Statistical Agency Addis Ababa, Ethiopia ORC Macro Calverton, Maryland, USA September, 2006: child vaccination coverage. Ethiopian Demographic and Health Survey,2005.
[12] Bizuhan A., Wale, Yibeltal T.and Bayou. Inequalities in Childhood Immunization in Ethiopia, 2011

[13] Belachew E.and Wakgari D. Factors associated with complete immunization coverage in children aged 12-23 months in Ambo Woreda, Central Ethiopia. BMC Public Health, 2012, 12:566.

[14] Henoke T, Amare D and Mirkuzie W. Predictors of defaulting from completion of child immunization in south Ethiopia. BMC Public Health, 2009; 9: 150.

[15] Abdulraheem I., Onajole A., Jimoh A. and Oladipo A. Reasons for incomplete vaccination and factors for missed opportunities among rural Nigerian children. Journal of Public Health and Epidemiology Vol. 3(4), pp.194-203, April 2011. 